\title{
Expulsion of disclinations in nematic liquid crystals
}

\author{
Paolo Biscari ${ }^{1}$ and Timothy J. Sluckin ${ }^{2}$ \\ ${ }^{1}$ Dipartimento di Matematica, Politecnico, Piazza Leonardo da Vinci 32, 20133 Milano (Italy) and Istituto \\ Nazionale di Fisica della Materia, Via Ferrata 1, 27100 Pavia (Italy). \\ ${ }^{2}$ Faculty of Mathematical Studies, University, Southampton SO17 1BJ (United Kingdom).
}

Date: 15 December 2001

2001 PACS: 61.30.Jf - Defects in liquid crystals

61.30.Hn - Surface phenomena: alignment, anchoring, etc.

83.80.Xz - Liquid crystals: nematic, cholesteric, smectic, discotic, etc.

Submitted to European Journal of Applied Mathematics

\begin{abstract}
We study the interactions between a nematic liquid crystal disclination and the surface of the half-space which bounds it. When strong anchoring conditions are applied on the boundary, we show how the biaxial core of the disclination affects the repulsive force that tends to drive the disclination away from the surface. If we replace the strong boundary conditions with an anchoring potential, the surface-disclination interaction depends on the surface extrapolation length. In particular, we show that the nematic may expel the disclination if the anchoring strength is below a critical value.
\end{abstract}

\section{Introduction}

A confined nematic liquid crystal is forced to display point or line singularities whenever the boundary conditions imposed on it are topologically nontrivial $[24,19,5]$. In particular, topologically stable line defects (commonly named disclinations) arise whenever the director $\mathbf{n}$ rotates an odd multiple of $\pi$ when we follow its continuous variation around the line. They are made possible by the $\mathbf{n} \leftrightarrow-\mathbf{n}$ symmetry that characterizes the nematic. The mere existence of disclinations induces a paradox in the Frank variational theory of nematic liquid crystals [17], since any director field representing a topologically stable disclination apparently possesses an infinite free-energy.

One way to avoid the divergence in the free-energy is to excise from the system a small volume including the singularity, and then derive the static and dynamic properties of the disclinations by taking the limit as the excised volume goes to zero [9]. Nevertheless, the complete physical description of small regions around the singularities requires an extension of Frank-Oseen-Zocher theory $[28,36,17]$, obtained by replacing the director order parameter with the tensor of secondorder moments of the local probability distribution of nematic molecules [12, 14]. As a first generalization of the classical theory, it has been shown that the structure and dynamical properties of point [30] and line [31,20] defects are deeply influenced by the reduction of the degree of orientation, even if one assumes that the nematic remains everywhere uniaxial, and that it becomes isotropic on the defect. Furthermore, numerical and theoretical studies $[23,35,6,25,26]$ of the core structure have proved that inside the core of a disclination the nematic not only decreases its degree of orientation, but it actually abandons the uniaxial phase, by becoming biaxial in a small, but finite, region surrounding the defect. Recently, tremendous interest in the structure and properties of liquid crystal disclinations has arisen, partly because they can be thought of as laboratory analogues of cosmological structures $[11,8]$, partly because despite their experimental visibility they are nevertheless extremely complex to describe.

When we consider a nematic disclination confined in a half-space where strong anchoring conditions are applied, the classical limit procedure described above (first applied by Eshelby [15]) 
yields a repulsive force simply proportional to the inverse of the distance between the line defect and the surface. On the other hand, in a recent investigation the usual strong anchoring conditions are replaced by an absolutely free boundary, where no anchoring is applied [34]. In this case the disclination is attracted towards the surface, and eventually expelled from the system.

In this paper we study the interaction between a $+\frac{1}{2}$ disclination and a planar surface, when either strong or weak anchoring conditions are imposed at the bounding surface, using the internal Landau-de Gennes potential to analyze the biaxial structure of the core of the disclination. In the former case, we find that if the disclination is sufficiently far away from the surface, the core radius tends to a constant, and the core structure gives no essential contribute to the repulsive force, so that we retrieve the classical results obtained in the limit of a vanishing core. Nevertheless, when the disclination approaches the surface, we find that the core radius shrinks, and the repulsive force increases, doubling its value with respect to the classical prediction.

Furthermore, when a weak anchoring potential is applied to the nematic, we show that the interaction between the surface and the disclination suffers deep modifications. If the anchoring is strong enough, the force is still repulsive at all distances, although it remains bounded even when the disclination approaches the surface. If however we decrease the anchoring strength, a critical distance $r_{\text {cr }}$ appears, such that the force is repulsive if the surface-disclination distance is greater than $r_{\mathrm{cr}}$, while it becomes attractive below $r_{\mathrm{cr}}$. The results obtained in [34] (attractive force at all distances) remain valid in the limiting case of vanishing anchoring at the boundary.

The paper is organized as follows. In Section 2 we describe the model and the free-energy functional we use. Section 3 is devoted to the strong anchoring limit; the surface-disclination force and the core structure will be analyzed therein. In Section 4 we replace the strong anchoring conditions by a weak anchoring potential and we analyze the qualitative changes suffered by the surface-disclination force. Finally, in Section 5 we discuss the results obtained.

\section{The model}

Let us consider a nematic liquid crystal confined in the half-space $\mathcal{B}=\left\{P=O+x \mathbf{e}_{x}+y \mathbf{e}_{y}+\right.$ $\left.z \mathbf{e}_{z}: x \geq 0\right\}$ with a $+\frac{1}{2}$ line defect, parallel to the $z$-direction and passing through the point $P_{\circ}=O+r \mathbf{e}_{x}$. In this section we introduce the free-energy functional and the boundary conditions that we will use to determine a quasi-equilibrium configuration for any given value of the distance $r$ between the disclination and the boundary of the nematic.

\subsection{Nematic order tensor}

We describe the local configuration of the nematic liquid crystal by means of the symmetric traceless tensor $\mathbf{Q}$ of second order moments of the local distribution of molecular orientations [12]. The local physical properties are linked to the degree of symmetry of $\mathbf{Q}$. The nematic is biaxial where the three eigenvalues of the order tensor are all different; it is uniaxial if two eigenvalues coincide; finally, it locally melts, and becomes isotropic, if $\mathbf{Q}$ is proportional to the identity tensor I. When the nematic is uniaxial, $\mathbf{Q}$ can be written as

$$
\mathbf{Q}=s\left(\mathbf{n} \otimes \mathbf{n}-\frac{1}{3} \mathbf{I}\right)
$$

where $s \in\left[-\frac{1}{2}, 1\right]$ is the degree of orientation [14] and the unit vector $\mathbf{n}$ is the director. In particular $s$, and thus $\mathbf{Q}$, vanishes if the nematic is isotropic.

We need to describe $a+\frac{1}{2}$ disclination parallel to the $z$-direction. The symmetry of $\mathcal{B}$ suggests to focus on distributions $\mathbf{Q}$ independent of $z$ with $\mathbf{e}_{z}$ as one of the eigenvalues

$$
\mathbf{Q}=\lambda_{1} \mathbf{e}_{1} \otimes \mathbf{e}_{1}+\lambda_{2} \mathbf{e}_{2} \otimes \mathbf{e}_{2}+\lambda_{z} \mathbf{e}_{z} \otimes \mathbf{e}_{z},
$$


where

$$
\left\{\begin{array}{l}
\mathbf{e}_{1}(x, y)=\cos \varphi(x, y) \mathbf{e}_{x}+\sin \varphi(x, y) \mathbf{e}_{y} \\
\mathbf{e}_{2}(x, y)=-\sin \varphi(x, y) \mathbf{e}_{x}+\cos \varphi(x, y) \mathbf{e}_{y}
\end{array} \quad \text { and } \quad \lambda_{z}(x, y)=-\lambda_{1}(x, y)-\lambda_{2}(x, y) \quad \forall(x, y),\right.
$$

so that we will simply refer our configurations to the half-plane $\mathcal{B}_{0}:=\mathcal{B} \cap\{z=0\}$.

\subsection{Free energy functional}

We determine the equilibrium configuration of the nematic liquid crystal by minimizing the bulk free-energy functional

$$
\mathcal{F}_{\mathrm{b}}[\mathbf{Q}]:=\int_{\mathcal{B}_{0}}\left(f_{\mathrm{el}}(\mathbf{Q}, \nabla \mathbf{Q})+f_{\mathrm{LdG}}(\mathbf{Q})\right) \mathrm{d} a
$$

subject to strong boundary conditions or completed with an anchoring energy functional, as we describe below. Using the 1-constant approximation for the elastic part of the free-energy density and the usual expression for the Landau-de Gennes potential [12], the bulk free-energy functional (2.2) can be written as

$$
\mathcal{F}_{\mathrm{b}}[\mathbf{Q}]:=\int_{\mathcal{B}_{0}}\left(\frac{\kappa}{2}|\nabla \mathbf{Q}|^{2}+a \operatorname{tr} \mathbf{Q}^{2}-b \operatorname{tr} \mathbf{Q}^{3}+c \operatorname{tr} \mathbf{Q}^{4}\right) \mathrm{d} a,
$$

where $\kappa$ is an elastic constant and, in the nematic phase, $a<0$, while $b, c>0$. We remark that a more detailed study of the problem, or the treatment of nematic materials with quite different splay, twist and bend moduli, requires a more specific expression for the elastic potential ${ }^{1}$. Furthermore, numerical simulations $[35,18]$ prove that the $z$-symmetry of the equilibrium configurations is broken when the elastic moduli are assumed different. Thus, our symmetrical setting is strictly related to the 1-constant approximation assumed in (2.3).

If $\mathbf{Q}$ is everywhere uniaxial, with constant degree of orientation $s \equiv s_{0}$, the Landau-de Gennes potential simply contributes with an additive constant, and the whole free energy functional (2.3) reduces to the classical 1-constant approximation to Frank's free energy functional [17]

$$
\mathcal{F}_{\mathrm{Fr}}[\mathbf{n}]:=\kappa s_{0}^{2} \int_{\mathcal{B}_{0}}|\nabla \mathbf{n}|^{2} \mathrm{~d} a=\kappa s_{0}^{2} \int_{\mathcal{B}_{0}}|\nabla \varphi|^{2} \mathrm{~d} a .
$$

It was first noted by Lyutsyukov [22] that the order-tensor description of nematic liquid crystals avoids the free-energy divergence, since $\mathbb{S}^{4}$ has no defect in $d=3$, whereas $\mathbb{P}^{2}$ does. Thus, on some length scale the $\mathbb{P}^{2}$ defect would relax to the $\mathbb{S}^{4}$ non-defect. To analyze this relaxation, Lyutsyukov made use of a constraint which has been widely used subsequently $[29,3,7]$. To introduce it, we recall that the parameter $b$ in (2.3) is usually much smaller than both $|a|$ and $c$. In fact, $b$ is responsible for the isotropic-nematic transition being first order, but it is well-known [12] that this transition is only weakly first order. Thus (see [7] for details) we can impose on $\mathbf{Q}$ the constraint

$$
\operatorname{tr} \mathbf{Q}^{2}=2\left(\lambda_{1}^{2}+\lambda_{1} \lambda_{2}+\lambda_{2}^{2}\right) \equiv-\frac{a}{c}=: \frac{2}{3} s_{0}^{2},
$$

where $s_{0} \in(0,1]$ represents the degree of orientation preferred in the bulk. The constraint $(2.5)$ automatically minimizes the second- and fourth-order terms in the Landau-de Gennes potential, so that they will be henceforth dropped from the bulk free energy. Recent numerical simulations [1] confirm the validity of the constraint (2.5) in the regime $T \ll T_{\mathrm{NI}}$, while it gives only qualitative understanding of the results close to the nematic-isotropic transition, where $a$ vanishes.

\footnotetext{
${ }^{1}$ See [21] for the more general rotationally-invariant elastic potential quadratic in the gradient of the order tensor and at most quadratic in $\mathbf{Q}$
} 
To implement (2.5) we introduce a scalar parameter $u(x, y)[7]$, in terms of which we write the eigenvalues of $\mathbf{Q}$ as:

$$
\lambda_{1}=-\frac{s_{0}}{3}\left(u-\sqrt{3-3 u^{2}}\right), \quad \lambda_{2}=-\frac{s_{0}}{3}\left(u+\sqrt{3-3 u^{2}}\right), \quad \lambda_{z}=\frac{2}{3} s_{0} u ;
$$

$u$ may attain all the values in $[-1,1]$; in particular, when $u=-\frac{1}{2}$ the nematic is uniaxial with degree of orientation $s_{0}$ and director $\mathbf{e}_{1}$, while when $u=-1$ the nematic is uniaxial with degree of orientation $-s_{0}$ and director $\mathbf{e}_{z}$.

To sum up, we are left with just two parameters to identify the order tensor $\mathbf{Q}$ : the angle $\varphi$ characterizing the eigendirections, and the scalar $u$, in terms of which all the eigenvalues can be determined.

\subsection{Anchoring}

We will assume that a homeotropic uniaxial anchoring is applied on the nematic at its boundary $\partial \mathcal{B}_{0}=\mathcal{B}_{0} \cap\{x=0\}$. Thus, when studying strong anchoring effects, we will enforce the boundary condition

$$
\mathbf{Q}(0, y)=\mathbf{Q}_{0}:=s_{0}\left(\mathbf{e}_{x} \otimes \mathbf{e}_{x}-\frac{1}{3} \mathbf{I}\right) \quad \text { for all } y \in \mathbb{R},
$$

that, in terms of $\varphi$ and $u$, is equivalent to require that

$$
\varphi(0, y)=\pi \quad \text { and } \quad u(0, y)=-\frac{1}{2} \quad \text { for all } \quad y \in \mathbb{R} .
$$

By contrast, the study of weak anchoring effects requires a relaxation of the boundary condition (2.7) by inserting in the free energy functional the anchoring energy

$$
\mathcal{F}_{\mathrm{S}}[\mathbf{Q}]:=\frac{w}{2} \int_{\partial \mathcal{B}_{0}} \operatorname{tr}\left[\left(\mathbf{Q}-\mathbf{Q}_{0}\right)^{2}\right] \mathrm{d} \ell .
$$

Expression (2.9) for the anchoring energy is the most sensible generalization [27] to the order tensor of the classical Rapini-Papoular anchoring energy [33]. Indeed, if we insert in (2.9) both $\mathbf{Q}_{0}$ as in (2.7) and a uniaxial order tensor $\mathbf{Q}=s_{0}\left(\mathbf{n} \otimes \mathbf{n}-\frac{1}{3} \mathbf{I}\right)$, we obtain

$$
\mathcal{F}_{\mathrm{RP}}[\mathbf{n}]=w s_{0}^{2} \int_{\partial \mathcal{B}_{0}}\left(1-\left(\mathbf{n} \cdot \mathbf{e}_{x}\right)^{2}\right) \mathrm{d} \ell
$$

Finally, in order to enforce a $+\frac{1}{2}$ disclination at the point $P_{\mathrm{o}}$, we restrict our attention to angular configurations $\varphi(x, y)$ satisfying the topological property

$$
\int_{\gamma}\left|\frac{d \varphi}{d \ell}\right| \mathrm{d} \ell=\pi
$$

around any closed curve $\gamma$, having natural parameter $\ell$ and enclosing the point $P_{\circ}$ in $\mathcal{B}_{0}$. Condition (2.10) ensures that the planar eigenvectors of $\mathbf{Q}$ complete a half-turn when we follow their continuous variation along $\gamma[5]$.

\subsection{Classical minimizer and beyond}

In the classical Frank theory, which we can easily retrieve in our formulation by enforcing $u \equiv-\frac{1}{2}$, the Euler-Lagrange equation associated with the functional $\mathcal{F}_{\text {Fr }}$ in $(2.4)$ simply reduces to the Laplace equation in the plane. The minimizing fields $\varphi$ are thus the harmonic functions which satisfy suitable boundary conditions [9]. In particular, by using an image method, it is possible to construct explicitly an harmonic function $\varphi$ satisfying both (2.8) and (2.10): let $P_{\circ}=O+r \mathbf{e}_{x}$ be 


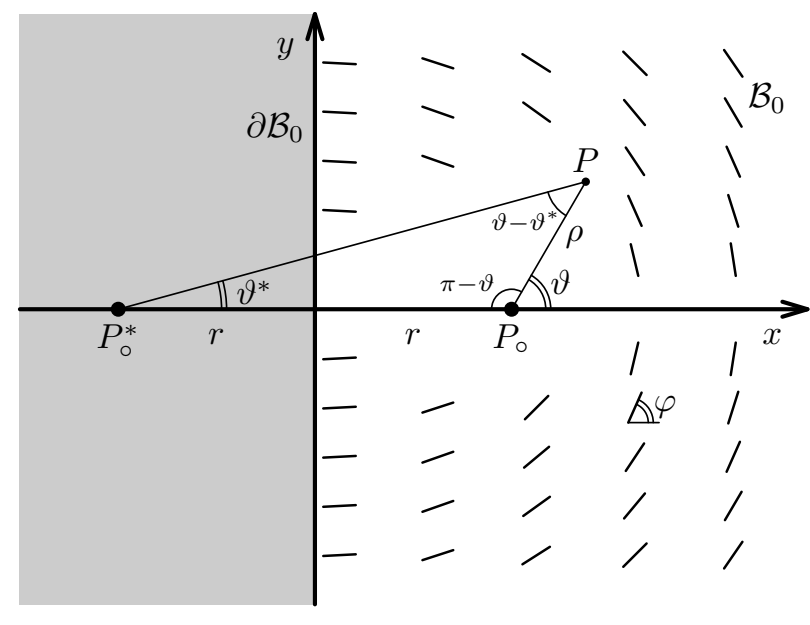

Figure 1: Geometry of the $+\frac{1}{2}$ line defect near a planar boundary, parallel to the disclination.

a point belonging to the disclination, and let $P_{\circ}^{*}=O-r \mathbf{e}_{x}$ be its mirror image with respect to the boundary $\partial \mathcal{B}_{0}$ (see Figure 1 ). Furthermore, for any point $P$, we introduce the angle $\vartheta$ determined by $\left(P-P_{\circ}\right)$ and $\mathbf{e}_{x}$, and the angle $\vartheta^{*}$ determined by $\left(P-P_{\circ}^{*}\right)$ and $\mathbf{e}_{x}$; then, a harmonic function satisfying both (2.8) and (2.10) is given by

$$
\varphi(x, y)=\frac{1}{2}\left(\pi+\vartheta(x, y)+\vartheta^{*}(x, y)\right)
$$

The well-known problem with the classical minimizer (2.11) is that it yields an infinite energy for any value of $r$. Nevertheless, it is possible to exclude from $\mathcal{B}_{0}$ a small disc $D_{\epsilon}$ of radius $\epsilon$, centered at $P_{\circ}$. The energy of the configuration (2.11) and thus the force acting on the disclination can then be computed. Finally one takes the $\epsilon \rightarrow 0$ limit of the force. As a result, one finds that $[15,10]$

$$
\mathbf{F}=\frac{\kappa \pi}{2 r} \mathbf{e}_{x} \quad \forall r>0
$$

To go beyond Frank's theory, we still keep the expression (2.11) for $\varphi$, but we relax the assumption $u \equiv-\frac{1}{2}$. This permit the nematic to decrease its degree of orientation, or even become biaxial. We expect the nematic to leave its most ordered state mainly close to the defect. We therefore parametrize the points in the plane by means of the angle $\vartheta$ introduced above, and their distance $\rho$ from $P_{\circ}$ (or, still better, by means of the dimensionless distance $\left.t:=\rho / r\right)$ :

$$
(P-O)=r(1+t \cos \vartheta) \mathbf{e}_{x}+r t \sin \vartheta \mathbf{e}_{y} .
$$

We then assume that $u$ depends on $t$, but not on $\vartheta$. When we want to enforce strong anchoring conditions on $\partial \mathcal{B}_{0}$, we must require the nematic to be uniaxial there. (The director orientation is automatically orthogonal to the surface as a result of $(2.11))$. This amounts to requiring $u(t) \equiv-\frac{1}{2}$ for all $t \geq 1$, which means that we assume that the minimizing distribution differs from the classical minimizer only inside a circle of radius $r$, centered at $P_{\circ}$.

If we introduce (2.6) and (2.11) in (2.1), and then the order tensor in (2.3), we obtain for the free-energy the expression

$$
\mathcal{F}_{\mathrm{b}}[u]=\frac{\kappa s_{0}^{2}}{3} \int_{0}^{T} t \mathrm{~d} t \int_{\cos \vartheta \geq-1 / t} \mathrm{~d} \vartheta\left[\frac{u^{\prime 2}}{1-u^{2}}+\frac{1-u^{2}}{t^{2}} \frac{1+2 t \cos \vartheta+t^{2}}{1+t \cos \vartheta+\frac{1}{4} t^{2}}+\frac{r^{2}}{\xi^{2}}\left(1-u\left(4 u^{2}-3\right)\right)\right]
$$


where $\xi:=\sqrt{\frac{3 \kappa}{2 b s_{0}}}$ is the nematic coherence length, $T:=R / r$, where $R$ is the dimension of the sample (which we will assume to be much greater than any other length that comes into play), and a constant has been added to the Landau-de Gennes potential in order to keep energies finite.

\section{Strong anchoring}

In this section we study the properties of the minimizers of the functional (2.13), subject to the condition $u(t) \equiv-\frac{1}{2}$ for all $t \geq 1$. Performing the integral in the angular variable in (2.13), we obtain

$$
\begin{aligned}
\mathcal{F}_{\mathrm{b}}[u]= & \frac{2 \pi}{3} \kappa s_{0}^{2} \int_{0}^{1}\left[\frac{t u^{\prime 2}}{1-u^{2}}+\frac{1-u^{2}}{t\left(1-\frac{t^{2}}{4}\right)}+\frac{r^{2}}{\xi^{2}} t\left(1-u\left(4 u^{2}-3\right)\right)\right] \mathrm{d} t \\
& +\frac{2}{3} \kappa s_{0}^{2} \int_{1}^{T}\left[\frac{3\left(t^{2}-2\right)}{t\left(t^{2}-4\right)} \arctan \left(\frac{t-2}{t+2} \sqrt{\frac{t+1}{t-1}}\right)+\frac{3}{2 t} \operatorname{arcos}\left(-\frac{1}{t}\right)\right] \mathrm{d} t \\
= & \frac{2 \pi}{3} \kappa s_{0}^{2} \int_{0}^{1}\left[\frac{t^{2} u^{\prime 2}}{1-u^{2}}+\frac{1-u^{2}}{1-\frac{t^{2}}{4}}+\frac{r^{2} t^{2}}{\xi^{2}}\left(1-u\left(4 u^{2}-3\right)\right)\right] \frac{\mathrm{d} t}{t} \\
& +\pi \kappa s_{0}^{2} \lg \frac{R}{r}+c_{1} \kappa s_{0}^{2}+\mathcal{O}\left(\frac{r}{R}\right), \\
\text { where } c_{1}:=\int_{1}^{\infty} & {\left[\frac{2\left(t^{2}-2\right)}{t\left(t^{2}-4\right)} \arctan \left(\frac{t-2}{t+2} \sqrt{\frac{t+1}{t-1}}\right)+\frac{1}{t} \operatorname{arcos}\left(-\frac{1}{t}\right)-\frac{\pi}{t}\right] \mathrm{d} t=-1.31474 \ldots }
\end{aligned}
$$

\subsection{Repulsive force on the disclination}

The Euler-Lagrange equation associated with the functional (3.1) is

$$
\frac{d}{d t}\left(\frac{2 t u^{\prime}}{1-u^{2}}\right)=\frac{2 t u^{\prime 2} u}{\left(1-u^{2}\right)^{2}}-\frac{2 u}{t\left(1-\frac{t^{2}}{4}\right)}+\frac{r^{2}}{\xi^{2}} t\left(3-12 u^{2}\right)
$$

together with the boundary conditions $u(1)=-\frac{1}{2}, u^{\prime}(0)=0$. It can be numerically solved using a relaxation method and yields the minimizing distribution for any value of $r$. Then, differentiating the resulting minimal free-energy with respect to $r$, we obtain the quasi-static elastic force acting on the disclination:

$$
\mathbf{F}=-\frac{d \mathcal{F}_{\min }}{d r} \mathbf{e}_{x}
$$

We remark that (3.3) is valid only as long as backflow effects can be neglected, that is, only when the translational degrees of freedom do not influence the rotation of the nematic molecules. In fact, the force acting on the disclination is exactly given by (3.3) in the limit of vanishing macroscopic velocities [10], so that we will often refer to the force (3.3) as a quasi-static force.

Before embarking upon an analysis of the numerical results, we focus on two asymptotic limits which can be studied analytically.

- When $r \gg \xi$ (large distance limit), the $r^{2} / \xi^{2}$ term dominates in (3.1). Therefore, and in order to make the coefficient of $r^{2} / \xi^{2}$ as small as possible, the solution of (3.2) remains almost constantly equal to $-\frac{1}{2}$ for all values of $t \gtrsim \xi / r$. Taking into account that the first two terms in the functional depending on $u$ are scale-invariant under the transformation 
$t \mapsto \alpha t$, while the third scales as $\alpha^{2}$, we then find

$$
\begin{aligned}
& \int_{0}^{1}\left[\frac{t^{2} u^{\prime 2}}{1-u^{2}}+\frac{1-u^{2}}{1-\frac{t^{2}}{4}}+\frac{r^{2} t^{2}}{\xi^{2}}\left(1-u\left(4 u^{2}-3\right)\right)\right] \frac{\mathrm{d} t}{t} \\
\simeq & \int_{0}^{\frac{\xi}{r}}\left[\frac{t^{2} u^{\prime 2}}{1-u^{2}}+\frac{1-u^{2}}{1-\frac{t^{2}}{4}}+\frac{r^{2} t^{2}}{\xi^{2}}\left(1-u\left(4 u^{2}-3\right)\right)\right] \frac{\mathrm{d} t}{t}+\int_{\frac{\xi}{r}}^{1}\left[\frac{1-\frac{1}{4}}{1-\frac{t^{2}}{4}}\right] \frac{\mathrm{d} t}{t} \\
= & \int_{0}^{1}\left[\frac{t^{2} u^{\prime 2}}{1-u^{2}}+\frac{1-u^{2}}{1-\frac{t^{2}}{4}}+t^{2}\left(1-u\left(4 u^{2}-3\right)\right)\right] \frac{\mathrm{d} t}{t}+\frac{3}{8} \log \frac{4 r^{2}-\xi^{2}}{3 \xi^{2}} .
\end{aligned}
$$

Thus, in this limit,

$$
\mathcal{F}_{\text {min }} \simeq \frac{\pi}{2} \kappa s_{0}^{2} \log \frac{R}{r \xi}+\mathcal{F}_{\text {core }}+\text { const. }+\mathcal{O}\left(\frac{\xi^{2}}{r^{2}}\right)+\mathcal{O}\left(\frac{r}{R}\right),
$$

where $\mathcal{F}_{\text {core }}$ denotes the elastic energy stored in the region $t \in[0,1]$ when $r \simeq \xi$. Using (3.4) we retrieve the classical result

$$
\mathbf{F}=\frac{\pi \kappa s_{0}^{2}}{2 r} \mathbf{e}_{x}\left(1+\mathcal{O}\left(\frac{\xi^{2}}{r^{2}}\right)+\mathcal{O}\left(\frac{r}{R}\right)\right) .
$$

Physically, the fact that $u$ becomes noticeably different from $-\frac{1}{2}$ only in the region $t \in\left[0, \frac{\xi}{r}\right]$ (that is, $\rho \in[0, \xi]$ ) implies that the dimension of the core tends to a finite value (closely related to the nematic coherence length) when the disclination is sufficiently far away from the surface. We emphasize that (3.5) is in perfect agreement with the classical result (2.12) if we neglect terms $\mathcal{O}\left(\xi^{2} / r^{2}\right)$, that is, if we neglect the core radius.

- By contrast, when $r \ll \xi$ (short distance limit), the term containing $r^{2} / \xi^{2}$ in (3.1) can be neglected, and $u$ leaves its bulk value $-\frac{1}{2}$ in the whole interval $t \in[0,1]$ (that is, $\rho \in[0, r]$ ). Consequently, we have

$$
\mathcal{F}_{\text {min }} \simeq \pi \kappa s_{0}^{2} \log \frac{R}{r}+\mathcal{F}_{\text {core }}+\text { const. }+\mathcal{O}\left(\frac{r^{2}}{\xi^{2}}\right)+\mathcal{O}\left(\frac{r}{R}\right),
$$

from which we find

$$
\mathbf{F}=\frac{\pi \kappa s_{0}^{2}}{r} \mathbf{e}_{x}\left(1+\mathcal{O}\left(\frac{r^{2}}{\xi^{2}}\right)+\mathcal{O}\left(\frac{r}{R}\right)\right)
$$

so that the coefficient of $r^{-1}$ in the repulsive force doubles at short distances.

Figure 2 illustrates the behaviour of the repulsive force acting on the disclination as a function of the distance $r$ from the boundary. The numerical results confirm the analytical limits described above, and give an estimate of the distance from the surface at which the intensity of the repulsive force matches the intensity computed from the classical model: when $r \gtrsim 3 \xi$ the classical model can be applied without any serious error.

\subsection{Core structure}

The degree of orientation of a nematic liquid crystal always decreases close to a defect line. Furthermore, in $[35,6]$ it has been proved that the nematic becomes biaxial inside the core of a disclination. The defect can be thought of as the region where the nematic is uniaxial with a negative degree of orientation, and is surrounded by a closed surface where one of the eigenvalues of the order tensor vanishes. Figure 3a, which exhibits the spatial variation of the eigenvalues of $\mathbf{Q}$ obtained through a numerical solution of (3.2), confirms this prediction. 


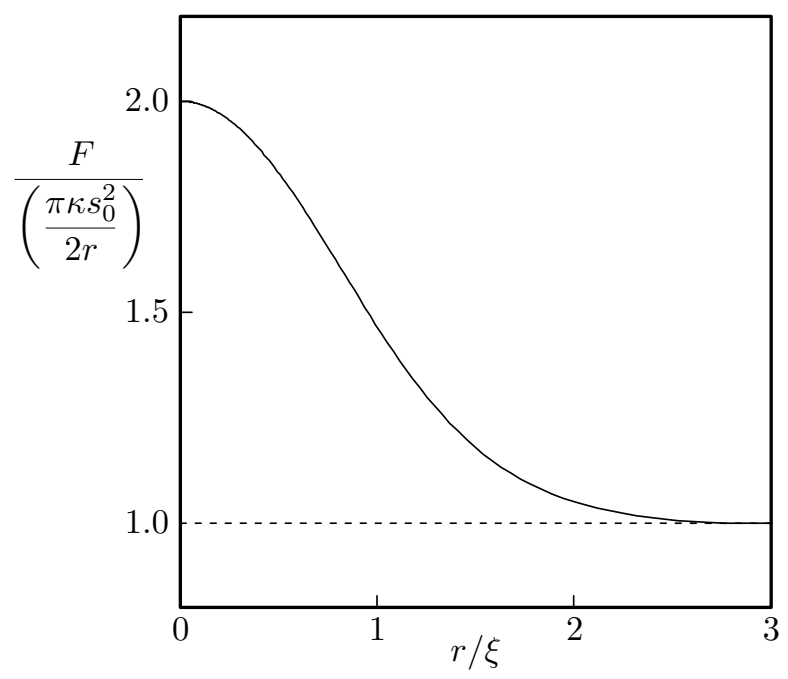

Figure 2: Intensity of the quasi-static force acting on a disclination placed at a distance $r$ from a boundary where strong anchoring is applied.

We can generalize the definition of degree of orientation to biaxial order tensors [4] as

$$
s\left(\lambda_{1}, \lambda_{2}, \lambda_{3}\right):=\left(\frac{27}{2} \prod_{i=1}^{3} \lambda_{i}\right)^{\frac{1}{3}} .
$$

Then, $s$ is positive in the bulk but negative inside the defect core. Furthermore, Figure $3 \mathrm{~b}$ shows that this transition is extremely sharp. It is thus physically as well as mathematically meaningful to define the core radius $\rho_{0}$ as the distance from the disclination at which one of the eigenvalues, and thus the degree of orientation, vanishes.

Figure 4 analyzes how the core radius depends on the distance of the disclination from the surface. In particular, it confirms the asymptotic analysis performed in $\S 3.1$. When the disclination approaches the surface, the core reduces its radius, scaling as $r$ when this latter vanishes (see Figure 4b). By contrast, when the defect moves inside the bulk, the core radius tends to a constant value (see Figure 4a). Furthermore, it is interesting to note that $\lim _{r \rightarrow 0} \frac{\rho_{0}}{r}=0.475 \ldots$ and $\lim _{r \rightarrow \infty} \frac{\rho_{0}}{\xi}=0.445 \ldots$, so that the core radius tends to assume a value which is almost exactly a given fraction (slightly smaller than $\frac{1}{2}$ ) of the smaller length of the problem, be it $r$ or $\xi$.

\section{Weak anchoring}

To study weak anchoring effects, we proceed as follows. First, we add to the free-energy functional the anchoring energy (2.9). Then, we modify the geometrical setting of Figure 1 by introducing the anchoring length $\xi_{a}$, similar to the cutoff length used in [16]. We assume (see Figure 5) that the configuration of a nematic subject to weak anchoring coincides with the configuration the same nematic would assume were strong anchoring conditions applied not at its surface $\partial \mathcal{B}_{0}$, but rather at a distance $\xi_{a}$ outside from the sample. Consequently, when $\xi_{a}$ increases, the elastic energy will in general decrease, since the nematic is allowed to abandon its homeotropic state at $\partial \mathcal{B}_{0}$, but at the same time the anchoring energy (2.9) will increase. Then, for any $r$ and any value of the surface extrapolation length, defined as $\zeta:=\kappa / w[12]$, we determine the optimal configuration by minimizing with respect to $\xi_{a}$ the complete free energy functional $\mathcal{F}:=\mathcal{F}_{\mathrm{b}}+\mathcal{F}_{\mathrm{s}}$. In general, the two surface lengths $\xi_{a}$ and $\zeta$ do not coincide [32], though they are closely related, as we describe below. 


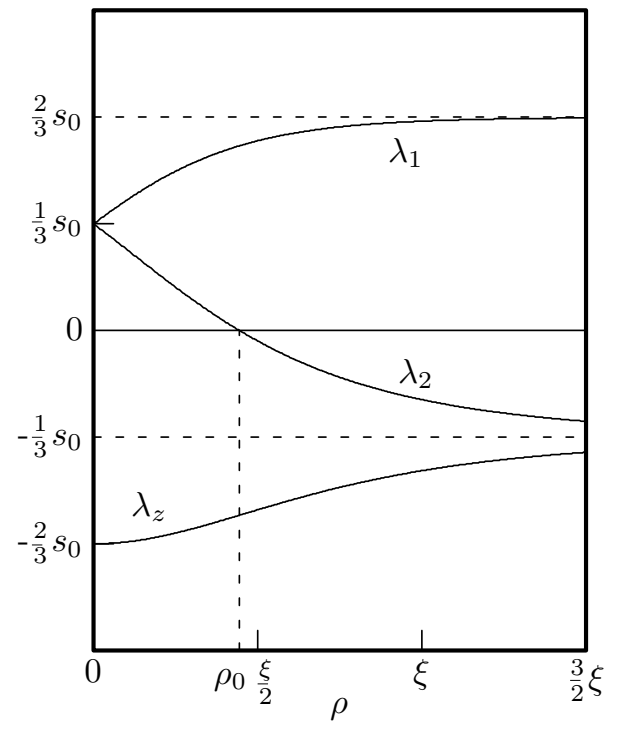

(a)

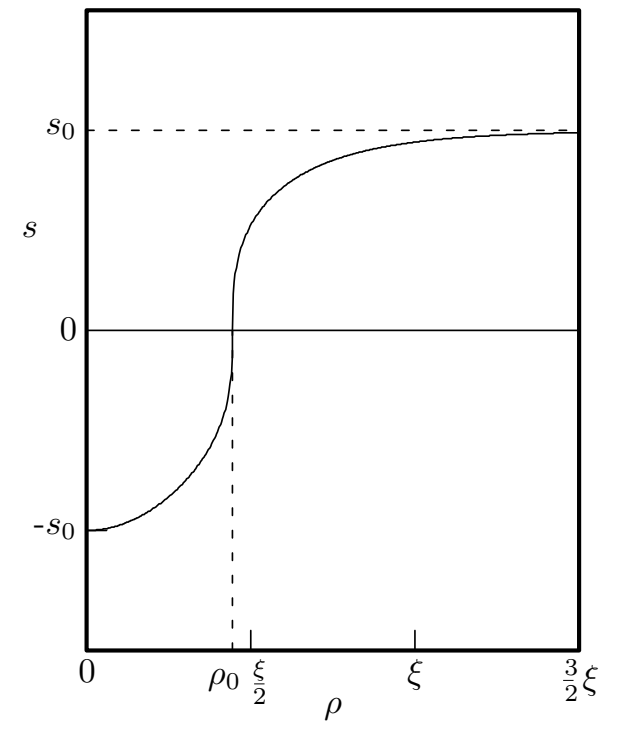

(b)

Figure 3: Structure of the core of a disclination. Fig. 3a shows the variation of the eigenvalues of the order tensor as a function of the distance from the center of the core, while Fig. 3b analyzes the behaviour of the degree of orientation. In both graphs, the disclination is placed at a distance $r=3 \xi$ away from the surface.

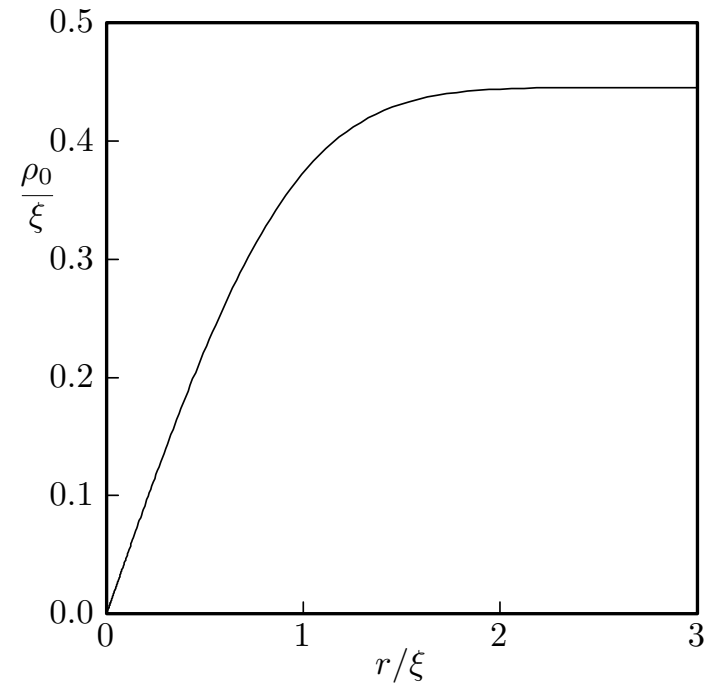

(a)

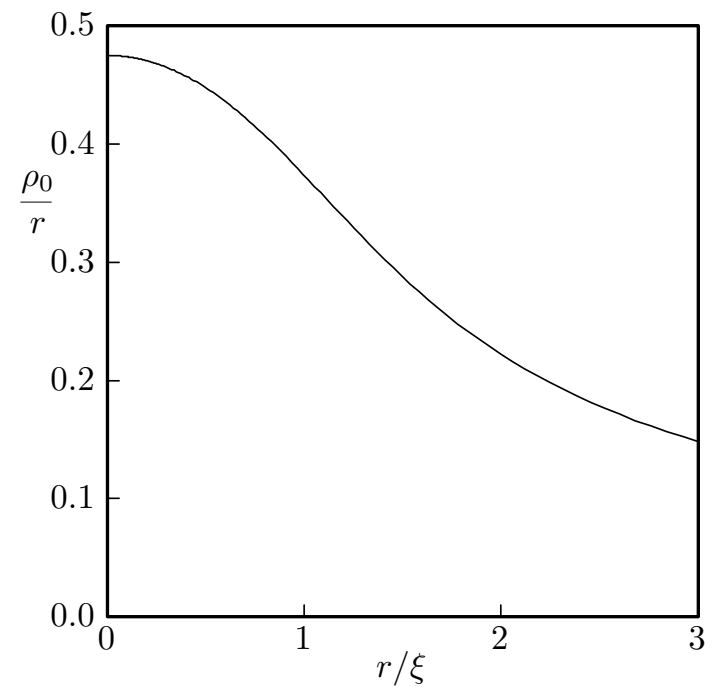

(b)

Figure 4: Core radius of a disclination line as a function of its distance from the surface. Fig. 4a shows that $\rho_{0}$ vanishes when the disclination approaches the surface, and tends to a constant value when the defect plunges into the bulk; Fig. 4b shows that $\rho_{0}=\mathcal{O}(r)$ when $r \rightarrow 0$. 


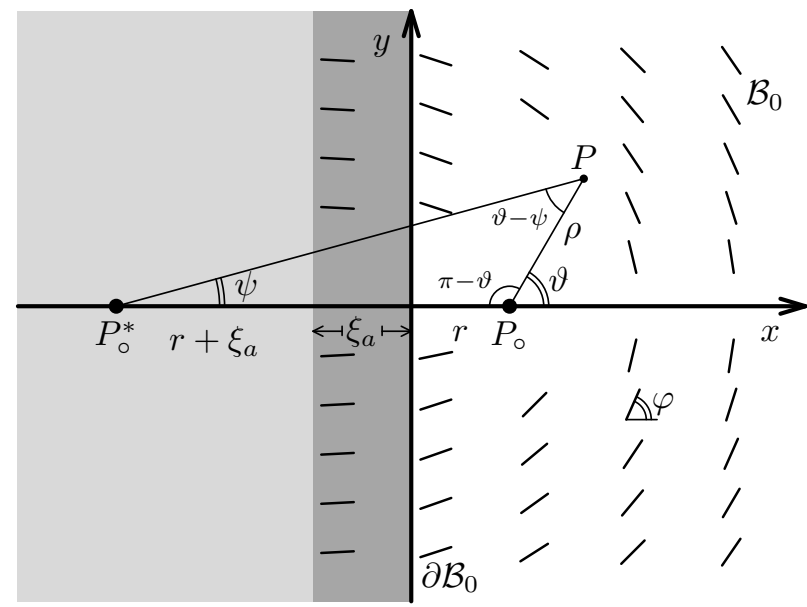

Figure 5: Geometrical setting for the modelling of a line disclination in a nematic liquid crystal with weak anchoring conditions applied at its surface.

This parametrization allows also for the description of the expulsion of the defect from the sample, since now $r$ is not forced to be positive. The only requirement to be imposed on the distance is that $r_{a}:=r+\xi_{a}$ must be positive, so that $r$ may become negative (thus representing an expelled defect) provided that the optimum $\xi_{a}$ is great enough.

When we insert the order tensors (2.7) and (2.1), with eigenvalues and eigenvectors given by (2.6) and (2.11), in the anchoring energy (2.9), we obtain:

$$
\begin{aligned}
\mathcal{F}_{\mathrm{S}} & =\frac{2}{3} \kappa s_{0}^{2} \frac{r_{a}}{\zeta} \int_{\tilde{r}}^{1} \frac{t}{\sqrt{t^{2}-\tilde{r}^{2}}}\left[2+u-\frac{\sqrt{3-3 u^{2}}\left(t^{2}+2 \tilde{r}-2 \tilde{r}^{2}\right)}{t \sqrt{t^{2}+4-4 \tilde{r}}}\right] \mathrm{d} t \\
& +\kappa s_{0}^{2} \frac{r_{a}}{\zeta} \int_{1}^{\frac{R}{r_{a}}} \frac{t}{\sqrt{t^{2}-\tilde{r}^{2}}}\left[1-\frac{t^{2}+2 \tilde{r}-2 \tilde{r}^{2}}{t \sqrt{t^{2}+4-4 \tilde{r}}}\right] \mathrm{d} t,
\end{aligned}
$$

where $\tilde{r}:=r / r_{a}$, while the bulk energy (2.3) becomes:

$$
\begin{aligned}
\mathcal{F}_{\mathrm{b}} & =\frac{2 \pi}{3} \kappa s_{0}^{2} \int_{0}^{\tilde{r}}\left[\frac{t u^{\prime 2}}{1-u^{2}}+\frac{1-u^{2}}{t\left(1-\frac{t^{2}}{4}\right)}+\frac{r_{a}^{2}}{\xi^{2}} t\left(1-u\left(4 u^{2}-3\right)\right)\right] \mathrm{d} t \\
& +\frac{2}{3} \kappa s_{0}^{2} \int_{\tilde{r}}^{1}\left[\frac{t u^{\prime 2}}{1-u^{2}}+\frac{r_{a}^{2}}{\xi^{2}} t\left(1-u\left(4 u^{2}-3\right)\right)\right] \operatorname{arcos}\left(-\frac{\tilde{r}}{t}\right) \mathrm{d} t \\
& +\frac{8}{9} \kappa s_{0}^{2} \int_{\tilde{r}}^{1}\left(1-u^{2}\right)\left[\frac{3\left(t^{2}-2\right)}{t\left(t^{2}-4\right)} \arctan \left(\frac{t-2}{t+2} \sqrt{\frac{t+\tilde{r}}{t-\tilde{r}}}\right)+\frac{3}{2 t} \operatorname{arcos}\left(-\frac{\tilde{r}}{t}\right)\right] \mathrm{d} t \\
& +\frac{2}{3} \kappa s_{0}^{2} \int_{1}^{\frac{R}{r_{a}}}\left[\frac{3\left(t^{2}-2\right)}{t\left(t^{2}-4\right)} \arctan \left(\frac{t-2}{t+2} \sqrt{\frac{t+\tilde{r}}{t-\tilde{r}}}\right)+\frac{3}{2 t} \operatorname{arcos}\left(-\frac{\tilde{r}}{t}\right)\right] \mathrm{d} t .
\end{aligned}
$$

\subsection{Asymptotic regimes}

Again, we will first analyze the functionals (4.1) and (4.2) in the asymptotic regimes representing a disclination very far from or very close to the surface. Then, a complete picture, based on numerical computations, will follow.

\subsubsection{Large distance limit $r \gg \xi, \xi_{a}, \zeta$}

The bulk free-energy $\mathcal{F}_{\mathrm{b}}$ can be computed as the free-energy corresponding to a defect placed at a distance $r_{a}$ from the surface minus the free-energy stored in the strip $x \in\left[-\xi_{a}, 0\right)$, which lies 
outside the system. Furthermore, the structure parameter $u$ can be assumed to be constantly equal to $-\frac{1}{2}$ in this strip, being in this limit the core region very far from the surface. Thus we obtain

$$
\begin{aligned}
\mathcal{F}_{\mathrm{b}} & =\frac{\pi}{2} \kappa s_{0}^{2} \log \frac{R}{r_{a} \xi}+\text { const. }+\mathcal{O}\left(\frac{\xi^{2}}{r^{2}}\right)+\mathcal{O}\left(\frac{r}{R}\right)-3 \kappa s_{0}^{2} \frac{\xi_{a}}{r} \int_{1}^{\infty} \frac{\sqrt{t^{2}-1}}{t^{3}} \mathrm{~d} t+\mathcal{O}\left(\frac{\xi_{a}^{2}}{r^{2}}\right) \\
& =\mathcal{F}_{\text {str }}-\frac{5 \pi}{4} \kappa s_{0}^{2} \frac{\xi_{a}}{r}+\mathcal{O}\left(\frac{\xi_{a}^{2}}{r^{2}}\right)+\mathcal{O}\left(\frac{\xi^{2}}{r^{2}}\right)+\mathcal{O}\left(\frac{r}{R}\right)
\end{aligned}
$$

where $\mathcal{F}_{\text {str }}$ denotes the free energy obtained in the strong anchoring case. Analogously, we obtain for the anchoring energy

$$
\mathcal{F}_{\mathrm{s}}=2 \kappa s_{0}^{2} \frac{\xi_{a}^{2}}{r \zeta} \int_{1}^{\infty} \frac{\sqrt{t^{2}-1}}{t^{3}} \mathrm{~d} t+\mathcal{O}\left(\frac{\xi_{a}^{3}}{r^{2} \zeta}\right)=\frac{\pi}{2} \kappa s_{0}^{2} \frac{\xi_{a}^{2}}{r \zeta}+\mathcal{O}\left(\frac{\xi_{a}^{3}}{r^{2} \zeta}\right)
$$

and thus

$$
\mathcal{F}=\mathcal{F}_{\text {str }}+\frac{\pi}{2} \kappa s_{0}^{2}\left(-\frac{5}{2} \frac{\xi_{a}}{r}+\frac{\xi_{a}^{2}}{r \zeta}\right)+\mathcal{O}\left(\frac{\xi_{a}^{2}}{r^{2}}\right)+\mathcal{O}\left(\frac{\xi^{2}}{r^{2}}\right)+\mathcal{O}\left(\frac{r}{R}\right)
$$

The dominant part in the functional (4.3) is minimized when

$$
\xi_{a}=\frac{5}{4} \zeta
$$

and its minimum value is

$$
\mathcal{F}_{\text {min }} \simeq \mathcal{F}_{\text {str }}-\frac{25 \pi}{32} \kappa s_{0}^{2} \frac{\zeta}{r}
$$

from which we obtain

$$
F(r)=-\frac{d \mathcal{F}_{\min }}{d r}=F_{\mathrm{str}}-\frac{25 \pi}{32} \kappa s_{0}^{2} \frac{\zeta}{r^{2}}+o\left(r^{-2}\right),
$$

where $F_{\text {str }}$ is the value of the quasi-static force when strong anchoring conditions are applied. Equation (4.6) shows that the correction to the quasi-static force tends to decrease its absolute value. Nevertheless, in this limit this correction is $\mathcal{O}\left(r^{-2}\right)$, and thus negligible if compared to $F_{\text {str }}$ itself, which is $\mathcal{O}\left(r^{-1}\right)$ as (3.5) shows.

\subsubsection{Short distance limit $r \ll \xi, \xi_{a}, \zeta$}

To analyze this case, we begin by estimating the value at which the anchoring length $\xi_{a}$ tends when $r \rightarrow 0$. In this case, the dominant contribution in (4.2) arises from the last integral, so that

$$
\mathcal{F}_{\mathrm{b}}=\pi \kappa s_{0}^{2} \log \frac{R}{\xi_{a}}+\mathcal{O}\left(\frac{\xi_{a}}{R}\right)+\mathcal{O}\left(\frac{\xi_{a}^{2}}{\xi^{2}}\right)
$$

since the last integrand in (4.2) behaves as $3 \pi /(2 t)$ when $t \gg 1$. For the anchoring energy we obtain:

$$
\begin{aligned}
\mathcal{F}_{\mathrm{S}} & =w s_{0}^{2} \xi_{a}\left[\frac{2}{3} \int_{0}^{1}\left(2+u-\frac{\sqrt{3-3 u^{2}} t}{\sqrt{t^{2}+4}}\right) \mathrm{d} t+\int_{1}^{\frac{R}{\xi_{a}}}\left(1-\frac{t}{\sqrt{t^{2}+4}}\right) \mathrm{d} t\right] \\
& =c_{2} w s_{0}^{2} \xi_{a}+\mathcal{O}\left(\frac{\xi_{a}}{R}\right),
\end{aligned}
$$

where $c_{2}:=\frac{2}{3} \int_{0}^{1}\left(2+u-\frac{\sqrt{3-3 u^{2}} t}{\sqrt{t^{2}+4}}\right) \mathrm{d} t+\sqrt{5}-1$. Thus,

$$
\mathcal{F}=\kappa s_{0}^{2}\left(\pi \log \frac{R}{\xi_{a}}+c_{2} \frac{\xi_{a}}{\zeta}\right)+\mathcal{O}\left(\frac{\xi_{a}}{R}\right)+\mathcal{O}\left(\frac{\xi_{a}^{2}}{\xi^{2}}\right)
$$


which is minimized when

$$
\xi_{a}=\frac{\pi}{c_{2}} \zeta,
$$

so that we again retrieve that the anchoring length scales with the surface extrapolation length, at least as long as this latter is small enough when compared to the nematic coherence length.

Now, in order to estimate the quasi-static force on the disclination when it reaches the surface, we must determine the leading terms in $r$ (when $r \rightarrow 0$ ) to (4.1) and (4.2). For the sake of brevity, we skip here the lengthy details of this computation, and we directly give the results:

$$
\mathcal{F}_{\mathrm{b}}=\left.\mathcal{F}_{\mathrm{b}}\right|_{r=0}+\left(c_{3}+c_{4} \frac{\xi_{a}^{2}}{\xi^{2}}\right) \kappa s_{0}^{2} \frac{r}{\xi_{a}}+\mathcal{O}\left(\frac{r^{2}}{\xi_{a}^{2}+\xi^{2}}\right),
$$

with $c_{3}:=\frac{2}{3} \int_{0}^{1}\left(\frac{t u^{\prime 2}}{1-u^{2}}+\frac{4\left(1-u^{2}\right)\left(1+t^{2}\right)}{t\left(4+t^{2}\right)}\right) \frac{\mathrm{d} t}{t}+\frac{1}{2}-2 \pi+\frac{3}{2} \arctan 2$, and $c_{4}:=\int_{0}^{1}\left(1-u\left(4 u^{2}-3\right)\right) \mathrm{d} t$.

On the other hand,

$$
\mathcal{F}_{\mathrm{s}}=\left.\mathcal{F}_{\mathrm{s}}\right|_{r=0}-c_{5} w s_{0}^{2} r+\mathcal{O}\left(\frac{r^{2}}{\xi_{a}}\right)
$$

with $c_{5}:=\log (2+\sqrt{5})+\frac{2}{5} \sqrt{5}-c_{2}-\frac{8}{3} \sqrt{3} \int_{0}^{1} \sqrt{1-u^{2}} \frac{\left(t^{2}+2\right) \mathrm{d} t}{t\left(t^{2}+4\right)^{3 / 2}}$.

The quasi-static force on a disclination placed exactly at the surface is thus

$$
F(0)=-\left(c_{3}+c_{4} \frac{\xi_{a}^{2}}{\xi^{2}}\right) \frac{\kappa s_{0}^{2}}{\xi_{a}}+c_{5} w s_{0}^{2}=\frac{\kappa s_{0}^{2}}{\zeta}\left[\left(c_{5}-\frac{c_{2} c_{3}}{\pi}\right)-\frac{\pi c_{4}}{c_{2}} \frac{\zeta^{2}}{\xi^{2}}\right] .
$$

The term $\left(c_{5}-c_{2} c_{3} / \pi\right)$ must clearly be (and turns out to be) positive, since the quasi-static force on the disclination must be repulsive and unbounded in the strong anchoring limit $\zeta \rightarrow 0$. Nevertheless, what it is really interesting is that both $c_{4}$ and $c_{2}$ are positive. The former, which measures the internal energy stored in the core, is so because we added a constant to the Landaude Gennes potential in (2.13) precisely to set the preferred degree of orientation as its zero-level. The latter measures the anchoring energy, apart from a multiplication factor (see (4.7)), and thus it is positive definite by construction (see (2.9)). Thus, the term within square brackets in (4.8) may change sign when the ratio $\zeta / \xi$ becomes great enough, so that the boundary force on the disclination may become attractive when the anchoring is sufficiently weak.

\subsection{Anchoring length}

Figure 6 illustrates how $\xi_{a}$ depends on $r$ for different values of the surface extrapolation length. The anchoring length increases when the defect approaches the surface, and it is almost proportional to the surface extrapolation length. This latter effect is enhanced when the ratio $\zeta / \xi$ becomes great enough (we recall $\zeta$ is typically greater than $\xi$ [2], in some cases even by more than one order of magnitude [13]). Furthermore, Figure 6 confirms the long-distance analytical prediction (4.4), and shows that when $\zeta$ becomes of the order of or greater than $\xi,(4.4)$ remains valid at all distances $r \geq 0$.

\subsection{Expulsion of the defect}

Figure 7 shows the fundamental qualitative changes that the weak anchoring induces in the quasistatic force acting on a disclination. In it, positive values of $F$ denote repulsion from the surface, while negative values indicate attraction. As long as $\zeta \lesssim \xi$, the force remains always repulsive, and it monotonically increases when the disclination approaches the surface, even if it tends to a finite limit when $r \rightarrow 0$. Furthermore, as we already noticed from (4.5), the long-distance behaviour of the quasi-static force does not depend on the anchoring strength. 


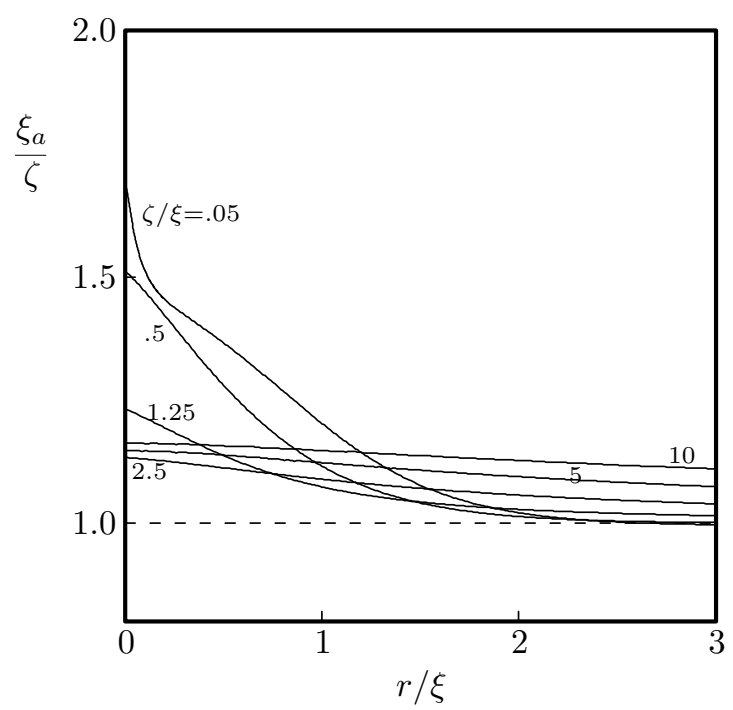

Figure 6: Dependence of the anchoring length $\xi_{a}$ on the distance between the disclination and the surface for the values of the surface extrapolation length indicated therein.

By contrast, and confirming (4.8), Figure 7 shows that when $\zeta$ increases (that is, the anchoring strength decreases) the quasi-static force may first lose its monotonicity, reaching its maximum when the line defect is at a finite distance from the surface. If the anchoring strength decreases further, the surface-disclination force becomes attractive at short-distances, that is, the sample tries to expel the disclination if it comes sufficiently close to the surface.

Figure 8a illustrates in detail the behaviour of $F(0)$ as a function of $\zeta$. The almost linear shape of the curve confirms (4.8). The critical value of the surface extrapolation length at which the surface force changes sign and the expulsion process may arise is given by $\zeta_{\mathrm{cr}}=.7974 \xi$.

Figure $8 \mathrm{~b}$ shows the $\zeta$-dependence of the critical distance $r_{\mathrm{cr}}$ at which the quasi-static force vanishes. The surface-disclination interaction is attractive when $r<r_{\mathrm{cr}}$ and repulsive at greater distances. It is worth noting that when $\zeta$ crosses $\zeta_{\mathrm{cr}}, r_{\mathrm{cr}}$ jumps abruptly from 0 to a value close to $0.13 \xi$. Furthermore, the dashed line shows that $r_{\mathrm{cr}}=\mathcal{O}(\zeta)$ when this latter diverges, so that in the weak anchoring limit we retrieve [34] that the quasi-static force on the disclination becomes attractive at all distances.

We remark that $r_{\mathrm{cr}}$ is always an unstable equilibrium distance for the disclination. The line defect is driven towards the bulk as long as it remains at a distance $r>r_{\mathrm{cr}}$. Nevertheless, if it crosses the critical distance from the surface, it is pushed outwards and eventually expelled from the sample.

\section{Concluding remarks}

We have studied the quasi-static force that a bounding surface induces on a nematic disclination. More precisely, we have treated in detail the case of a single $+\frac{1}{2}$ defect. However, the methods presented here can also be used to study the boundary interactions of disclinations of greater topological charges or the interactions between two or more defect lines. Our main outcomes are the following.

- When strong anchoring is enforced, the inclusion of a finite biaxial core in the description of the disclination increases the repulsive force that drives the disclination away from the surface. Nevertheless, this strengthening of the repulsive force is a short-range effect, that can be felt only when the distance between the disclination and the surface is of the order of the nematic coherence length. 


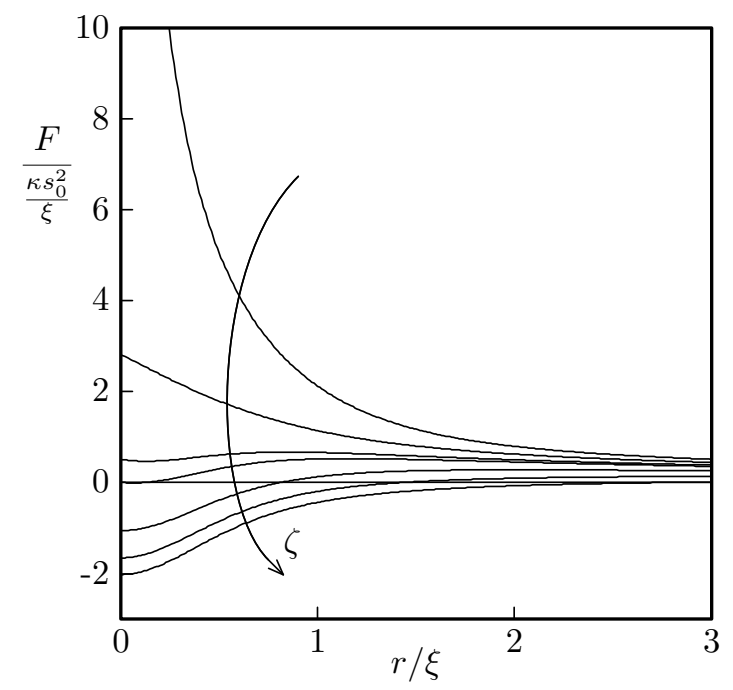

Figure 7: Quasi-static force acting on a disclination line placed at a distance $r$ from a surface where weak anchoring has been applied. From top to bottom, the graphs refer to the following values of the ratio between the surface extrapolation length $\zeta$ and $\xi: .05, .5,1,1.25,2.5,5$, and 10 .

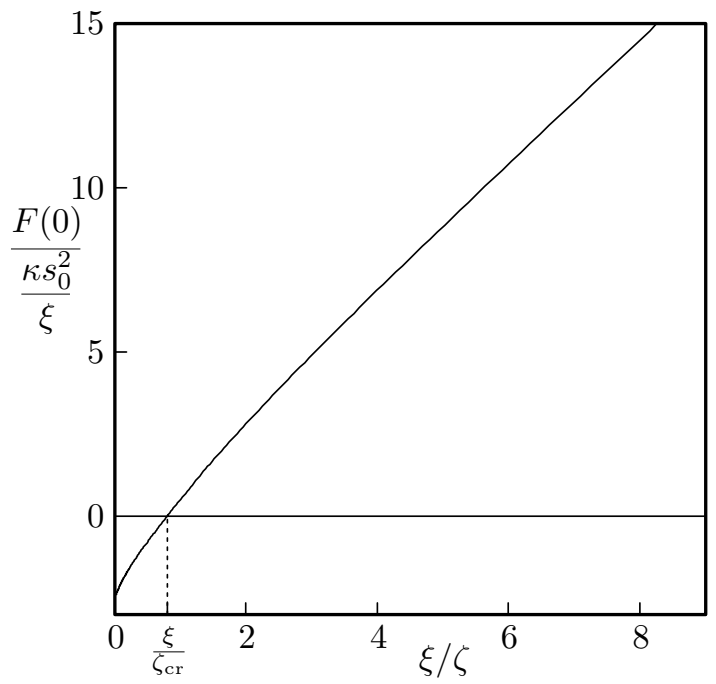

(a)

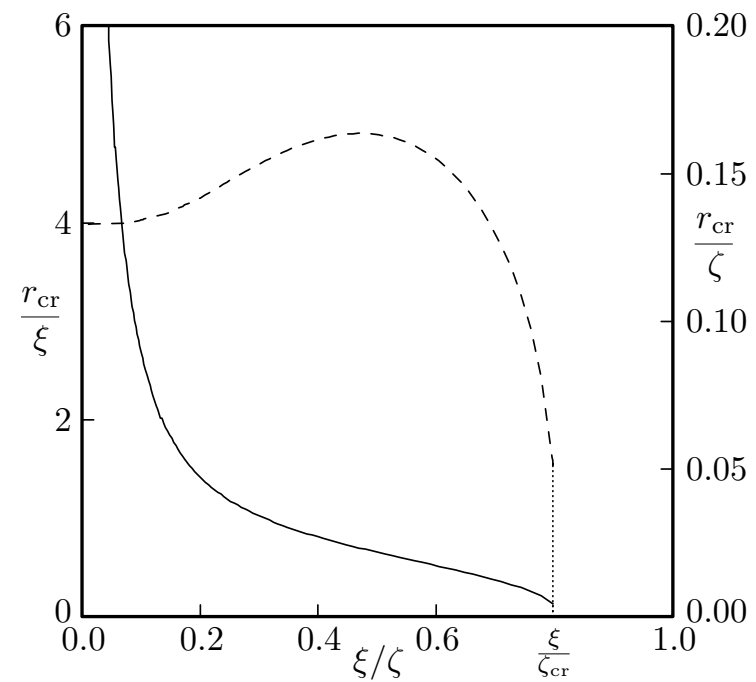

(b)

Figure 8: (a) Force on a disclination laying on the surface of a nematic liquid crystal as a function of the ratio between the nematic coherence length and $\xi$ and surface extrapolation length $\zeta$. Positive values of $F(0)$ imply that the disclination is pushed towards the nematic bulk, while negative values denote an expulsion of the disclination.

(b) Critical value of the distance from the surface at which the quasi-static force on the disclination vanishes, in units of $\xi$ (solid line) and $\zeta$ (dashed line). Both graphs are shown as a function of the ratio $\xi / \zeta$. 
- The core radius is almost constant (and closely related to the nematic coherence length) as long the disclination is sufficiently apart from the external surface; otherwise, it scales with the distance from the boundary.

- If we replace the strong anchoring conditions with a weak anchoring energy, a critical distance $r_{\mathrm{cr}}$ arises as soon as the the surface extrapolation length $\zeta$ becomes greater than the nematic coherence length. The surface-disclination interaction is now attractive for $r<r_{\mathrm{cr}}$, but it remains repulsive when $r<r_{\mathrm{cr}}$.

- The critical distance $r_{\mathrm{cr}}$ is proportional to $\zeta$ when this latter diverges. To be more precise, and considering that physically reasonable values for $\zeta$ lie in the range $10^{-9} \div 10^{-6} \mathrm{~m}$., that is $\zeta=1 \div 10^{2} \xi$, we obtain from the dashed line in Figure $8 \mathrm{~b}$ the estimate $r_{\mathrm{cr}}=.13 \zeta$.

Acknowledgements. This work has been supported by the Brite-EuRam Contract BRRTCT97-5003 Liquid crystals: surface properties from basic to applications. P.B. acknowledges the hospitality of the University of Southampton, where part of this work was carried out.

\section{References}

[1] D. Andrienko. On the theory and simulation of confined liquid crystals. PhD Thesis. University of Bristol, U.K. (2001).

[2] D. Andrienko, G. Germano, and M.P. Allen. Liquid crystal director fluctuations and surface anchoring by molecular simulation. Phys. Rev. E 62 (2000), 6688-6693.

[3] P. Biscari. Biaxial Persistence Length in Nematic Liquid Crystals. Mol. Cryst. Liq. Cryst. 290 (1996), 149-154.

[4] P. Biscari, G. Capriz, and E.G. Virga: Biaxial Nematic Liquid Crystals. In "BoundaryValue Problems for Partial Differential Equations and Applications", RMA Res. Notes Appl. Math. 29 (1993), 313-318.

[5] P. Biscari and G. Guidone Peroli. A Hierarchy of Defects in Biaxial Nematics. Comm. Math. Phys. 186 (1997), 381-392.

[6] P. Biscari, G. Guidone Peroli, and T.J. Sluckin. The topological microstructure of defects in nematic liquid crystals. Mol. Cryst. Liq. Cryst. 292 (1997), 91-101.

[7] P. Biscari and E.G. Virga. A Surface-induced Transition in Polymeric Nematics. Liq. Cryst. 22 (1997), 419-425.

[8] M.J. Bowick, L. Chandar, E.A. Schiff, and A.M. SRivastava. The cosmological Kibble mechanism in the laboratory: string formation in liquid crystals. Science 263 (1994), 943-945.

[9] H. Brezis, J.M. Coron, and E. Lieb. Harmonic maps with defects. Comm. Math. Phys. 107 (1986), 649-705.

[10] P. Cermelli and E. Fried. The evolution equation for a disclination in a nematic fluid. Preprint (2001).

[11] I. Chuang, R. Durrer, N. Turok, and B. Yurke. Cosmology in the laboratory: defect dynamics in liquid crystals. Science 251 (1991), 1336-1342.

[12] P.G. DE Gennes and J. Prost. The Physics of Liquid Crystals, Ed. Clarendon Press, Oxford (1993).

[13] I. Dozov and G. Durand. Surface controlled nematic bistability. Liq. Cryst. Today 8 (1998), $1-7$. 
[14] J. ERICKSEn. Liquid crystals with variable degree of orientation. Arch. Rat. Mech. Anal. 113 (1989), 97-120.

[15] J.D. Eshelby. The force on a disclination in a liquid crystal. Phil. Mag. A 42 (1980), 359-367.

[16] J.-B. Fournier and P. Galatola. Effective anchoring and scaling in nematic liquid crystals. Eur. Phys. Jnl. E 2 (2000), 59-65.

[17] F.C. Frank. On the theory of liquid crystals. Discuss. Far. Soc. 25 (1958), 19-28.

[18] S.D. Hudson and R.G. Larson. Monte Carlo simulation of a disclination core in nematic solutions of rod-like molecules. Phys. Rev. Lett. 70 (1993), 2916-2919.

[19] M. KlÉman. Defects in liquid crystals. Rep. Prog. Phys. 52 (1989), 555-654.

[20] G. KURZ. Hydrodynamics of defects in the abelian-Higgs model: an application to nematic liquid crystals. Ann. Phys. 282 (2000), 1-30.

[21] L. Longa, D. Monselesan, and H.-R. Trebin. An extension of the Landau-de Gennes theory for liquid crystals. Liq. Cryst. 2 (1987), 769-796.

[22] I.F. Lyuksyutov. Topological instability of singularities at small distances in nematics. Sov. Phys. JETP 48 (1978), 178-179.

[23] S. Meiboom, M. Sammon, and W.F. Brinkman. Lattice of disclinations: the structure of the blue phases of cholesteric liquid crystals. Phys. Rev. A 27 (1983), 438-454.

[24] N.D. Mermin. The Topological Theory of Defects in Ordered Media. Rev. Mod. Phys. 51 (1979), 591-648.

[25] N.J. Mottram and S.J. Hogan. Disclination core structure and induced phase change in nematic liquid crystals. Philos. Trans. Roy. Soc. London A 355 (1997), 2045-2064.

[26] N.J. Mottram and T.J. Sluckin. Disclination cores during the nematic to isotropic phase change. Liq. Cryst. 27 (2000), 1301-1304.

[27] M. Nobili and G. DuRAnd. Disorientation-induced disordering at a nematic-liquid-crystalsolid interface. Phys. Rev. A 46 (1992), R6174-R6177.

[28] C.W. Oseen. The theory of liquid crystals Trans. Far. Soc. 29 (1933), 883-900.

[29] E. Penzenstadler and H.-R. Trebin. Fine structure of point defects and soliton decay in nematic liquid crystals. Jnl. Phys. (France) 50 (1989), 1027-1040.

[30] L.M. Pismen and B.Y. Rubinstein. Motion of interacting point defects in nematics. Phys. Rev. Lett. 69 (1992), 96-99.

[31] L.M. Pismen and J. Rubinstein. Dynamics of disclinations in liquid crystals. Q.ly Appl. Math. 50 (1992), 535-545.

[32] N. Priezjev and R.A. Pelcovits. Surface extrapolation length and director structures in confined nematics. Phys. Rev. E 62 (2000), 6734-6738.

[33] A. Rapini and M. Papoular. Distorsion d'une lamelle nématique sous champ magnétique. Conditions d'ancrage aux parois. Jnl. Phys. Colloque C4 30 (1969), 54-56.

[34] G. Richardson. Line disclination dynamics in uniaxial nematic liquid crystals. Q.ly Jnl. Mech. Appl. Math. 53 (2000), 49-71.

[35] N. Schopohl and T.J. Sluckin. Defect core structure in nematic liquid crystals. Phys. Rev. Lett. 59 (1987), 2582-2584. 
[36] H. Zocher. The effect of a magnetic field on the nematic state. Trans. Far. Soc. 29 (1933), $945-57$. 